\title{
Compact $\mathrm{H}$ I clouds at high forbidden velocities in the inner Galaxy
}

\author{
J. M. Stil ${ }^{1}$ \\ Felix. J. Lockman ${ }^{2}$ \\ A. R. Taylor ${ }^{1}$ \\ J. M. Dickey ${ }^{3,4}$ and D. W. Kavars ${ }^{3}$ \\ P. G. Martin, T. A. Rothwell, and A. Boothroyd ${ }^{5}$ \\ N. M. McClure-Griffiths ${ }^{6}$
}

\begin{abstract}
The VLA Galactic Plane Survey (VGPS) of the first Galactic quadrant was searched for H I emission with velocities well above the maximum velocity allowed by Galactic rotation. A sample of 17 small fast-moving clouds was identified. The distribution of the ensemble of clouds in longitude and velocity indicates that the clouds are part of the Galactic disk, despite their large forbidden velocity. The median angular diameter of the clouds detected in the VGPS is 3.4. These clouds would not be noticed in previous low resolution surveys because of strong beam dilution. Assuming each cloud is located at the tangent point, a median cloud has a diameter of $10 \mathrm{pc}$, H I mass of $60 M_{\odot}$, and a velocity more than $25 \mathrm{~km} \mathrm{~s}^{-1}$ beyond the local terminal velocity derived from ${ }^{12} \mathrm{CO}$ observations. Three clouds
\end{abstract}

\footnotetext{
${ }^{1}$ Department of Physics and Astronomy, University of Calgary, 2500 University Drive N.W., Calgary AB T2N 1N4, Canada

${ }^{2}$ National Radio Astronomical Observatory, P.O. Box 2, Green Bank, West Virginia 24944, USA

${ }^{3}$ Department of Astronomy, University of Minnesota, 116 Church Street, SE, Minneapolis, MN 55455, USA

${ }^{4}$ School of Mathematics and Physics - Private Bag 37, University of Tasmania, Hobart, TAS 7001, Australia

${ }^{5}$ Department of Astronomy and Astrophysics, University of Toronto, 60 St. George Street Room 1403, Toronto ON, M5S 3H8, Canada

${ }^{6}$ Austalia Telescope National Facility, CSIRO, P.O. Box 76, Epping NSW 1710, Australia
} 
in the sample have a velocity between 50 and $60 \mathrm{~km} \mathrm{~s}^{-1}$ in excess of the local terminal velocity. The longitude distribution of the sample peaks near $l=30^{\circ}$, while the latitude distribution of the clouds is nearly flat. The observed longitude and latitude distributions are compared with simulated distributions taking into account the selection criteria of the cloud search. It is found that the number of clouds declines with distance from the Galactic center, with an exponential scale length $2.8-8 \mathrm{kpc}$ at the $99 \%$ confidence level. We find a lower limit to the scale height of the clouds of $180 \mathrm{pc}(\mathrm{HWHM})$, but the true value is likely significantly higher.

Subject headings: ISM: clouds — ISM: kinematics and dynamics — ISM: structure - Galaxy: disk

\section{Introduction}

A large fraction of the neutral halo in the inner Galaxy is in the form of clouds, observed in H I emission to at least $|z|=1.5 \mathrm{kpc}$ from the Galactic plane, and downward to within $|z| \approx 200 \mathrm{pc}$, where they become heavily blended with disk H I (Lockman 2002). A median halo cloud from a sample of 40 located at a median distance from the plane of $z=-940 \mathrm{pc}$ has an HI mass of $50 M_{\odot}$, a diameter of a few tens of parsecs, a column density of a few times $10^{19} \mathrm{~cm}^{-2}$, and average H I density of a few tenths $\mathrm{cm}^{-3}$. The clouds in the halo follow Galactic rotation, but with a fairly large cloud-to-cloud velocity dispersion of several tens of $\mathrm{km} \mathrm{s}^{-1}$ (Lockman 2002, 2003).

It is possible that the halo clouds are part of a widespread population, which may include the 'fast' clouds seen in optical and H I absorption surveys (e.g., Münch \& Zirin 1961, Radhakrishnan \& Srinivasan 1980, Mohan et al. 2004, Welsh, Sallmen \& Lallement 2004, Dwarakanath 2004) at high galactic latitude, and, when unresolved, as broad components in H I emission lines (Kulkarni \& Fich 1985; Kalberla et al. 1998), but see Dwarakanath et al. (2004) for a different interpretation of broad absorption features toward SGR A*. Kulkarni \& Fich (1985) constrained the the mass of a population of fast-moving clouds with a large scale height to at most $20 \%$ of the Galactic H I mass, but noted that it could contain most of the kinetic energy in the interstellar medium.

The clouds, however, may not be confined to the halo: Lockman \& Stil (2003) reported the discovery of a small H I cloud in the VLA Galactic Plane Survey (VGPS) (Taylor et al. 2002) which lies only 22 pc from $b=0^{\circ}$, but which shares many characteristics of the halo clouds. They speculated that it might be part of the halo population, visible at low latitude 
only because its random velocity puts it more than $40 \mathrm{~km} \mathrm{~s}^{-1}$ beyond the bulk of Galactic H I. The VGPS is unrivaled for study of low latitude Galactic H I in the first longitude quadrant. In this paper we present the results of a systematic search for clouds with velocities beyond that permitted by normal Galactic rotation at the low Galactic latitudes covered by this new survey.

\section{Data Set and Search parameters}

The VLA Galactic Plane Survey (Taylor et al. 2002) combines observations of the 21 $\mathrm{cm} \mathrm{H}$ I line and continuum emission over Galactic longitudes $18^{\circ} \leq l \leq 67^{\circ}$ made with the D configuration of the Very Large Array (VLA) and the Green Bank Telescope (GBT) of the $\mathrm{NRAO}^{1}$. The survey covers Galactic latitudes \pm 1.3 at low longitudes to \pm 2.3 at the higher longitudes (Taylor et al. 2002). The resolution of the VGPS is $1^{\prime} \times 1^{\prime} \times 1.56 \mathrm{~km} \mathrm{~s}^{-1}(\mathrm{FWHM})$ in the $21 \mathrm{~cm}$ line and spectra extend over $240 \mathrm{~km} \mathrm{~s}^{-1}$ at the full sensitivity of $2 \mathrm{~K}$ (r.m.s.) per channel. This is sufficient to reach velocities well beyond that of the H I emission at the higher longitudes. Higher velocities were covered by only one receiver channel in the VLA data. These data were also imaged, extending the velocity range by $\sim 30 \mathrm{~km} \mathrm{~s}^{-1}$, although at a sensitivity degraded by a factor $\sqrt{2}$.

In the inner Galaxy the maximum permitted rotational velocity at any longitude can be established from observations of H I or molecular clouds (e.g. Burton \& Gordon 1978, Clemens 1985) In general there is a well-defined terminal velocity at any $|l|<90^{\circ}$ : for a flat rotation curve $V_{\tan }=V_{\odot}(1-\sin l)$. Random motions can carry individual clouds to 'forbidden' velocities $V_{\mathrm{LSR}}>V_{\mathrm{tan}}$. For example, some halo clouds have random velocities in excess of $40 \mathrm{~km} \mathrm{~s}^{-1}$ (Lockman 2002, 2003).

Clouds at positive forbidden velocities in the VGPS were identified by visual inspection of the data both at the full angular resolution, and also smoothed to 2 ' resolution to improve the sensitivity to resolved clouds of low brightness. The data were also searched by applying a spectral filter. Beyond velocity $V_{2 \mathrm{~K}}$, defined as the velocity where $T_{\mathrm{b}}=2 \mathrm{~K}$ on the positive velocity wing of the H I profiles, a search was done for occurrences of 5 consecutive channels with brightness temperature exceeding 3 times the r.m.s. noise. The velocities searched correspond to $V_{\mathrm{LSR}}>V_{2 \mathrm{~K}} \approx V_{\mathrm{tan}}+25 \mathrm{~km} \mathrm{~s}^{-1}$. The images resulting from the spectral filtering were inspected for cloud candidates missed in the visual examination of the data. The final sample of clouds is believed to be complete for velocities exceeding $V_{2 \mathrm{~K}}$ and peak

\footnotetext{
${ }^{1}$ The National Radio Astronomy Observatory is operated by Associated Universities, Inc., under a cooperative agreement with the National Science Foundation.
} 
H I brightness temperatures $\gtrsim 10 \mathrm{~K}$. Lines-of-sight directly toward very bright continuum sources were not included in the search because of the possibility of systematic effects in the H I spectra.

Some cloud candidates detected in the VLA data were confirmed using the VGPS zerospacing dataset from the Green Bank Telescope (GBT) and also by analyzing subsets of the VLA data taken over several different days. Fifteen cloud candidates were subsequently reobserved with the GBT in October 2004 during which H I spectra were taken at integration times up to one minute in the direction of the clouds and at $\pm 10^{\prime}$ offsets in $l$ and $b$. These deeper GBT spectra have typical rms noise levels of $<60 \mathrm{mK}$.

\section{Cloud Properties}

Table 1 lists observed properties of the forbidden velocity HI clouds which we have identified. The velocity, peak brightness temperature and line width $\Delta v$ (FWHM) were measured by fitting a Gaussian to the line profile through the brightest part of the cloud. The errors listed for these quantities are $1 \sigma$ errors from the Gaussian fits. The peak H I column density, $N_{\mathrm{HI}}$, was derived assuming that the lines are optically thin, a good assumption unless their excitation temperature is $<100 \mathrm{~K}$ (see below for limits on the temperature). Cloud diameters were determined by fitting an ellipse to the half peak $N_{\text {HI }}$ contour. The location of the clouds in longitude and velocity is shown in Figure 1. The upper boundary of the velocity range searched is indicated by a dashed line. Detected clouds fall in a band in Figure 1 which has a width of $\sim 60 \mathrm{~km} \mathrm{~s}^{-1}$ and follows the variation of the terminal velocity with longitude. The upper boundary of the velocity range of the ensemble of clouds does not appear to be limited by the velocity coverage of the survey. The fact that the velocities of the clouds change systematically with longitude following the main H I emission indicates that these clouds, like H I clouds in the lower halo, have kinematics dominated by normal Galactic rotation despite their large random velocities (Lockman 2002). These clouds should be considered as a disk population.

The peak brightness temperatures of clouds in the sample lie in the range 10 to $22 \mathrm{~K}$. This range is limited by sensitivity on the low end, and possibly by resolution on the high end, as some clouds in the sample have angular diameters comparable to the $1^{\prime}$ resolution.

The sensitive GBT spectra provided independent confirmation of the clouds in the sample. H I line profiles from the VGPS are shown in Figure 2 with the H I profiles from the follow-up observations made with the GBT. Figure 3 shows enlargements of the profiles of four clouds which are difficult to discern Figure 2, in conjunction with the mean of the 
profiles in the off-cloud positions. A significant excess over the mean off-cloud profile at the velocity of the VGPS cloud is seen in all profiles except for the cloud $42.46+1.31+105$ which suffers from inconsistencies between the profiles at the offset positions. The shoulder in the GBT profile of this cloud provides a tentative detection in the GBT data.

The median size of clouds in the present sample is 3.4 , resulting in a significantly lower peak brightness temperature in the GBT spectra because of beam dilution. The peak brightness temperature in the GBT profiles is less than $3 \mathrm{~K}$, which is much smaller than the $\sim 15 \mathrm{~K}$ peak brightness temperature at $1^{\prime}$ resolution through the brightest part of the clouds. The VGPS data suggest that values of $N_{\mathrm{HI}}$ for these clouds derived from GBT data alone would be underestimated by a factor $\sim 7$ in the median, and values of $\left\langle n_{\mathrm{HI}}\right\rangle$ by a factor $\sim 20$. A clear example is $25.48+0.16+165$. The peak brightness temperature in the GBT profile is a factor 10 smaller than in the VGPS profile, consistent with beam dilution of the $3^{\prime}$ diameter cloud.

The mean GBT spectra of the off positions were also inspected for evidence of an extended $\left(>10^{\prime}\right)$, low-column density component with the same velocity as the clouds identified in the VGPS. The sensitivity of this experiment is determined by the presence of a faint wing of the Galactic line profile which appears as a continuation of the line profile of emission at lower, permitted, velocities as shown in Figure 2 and Figure 3. No evidence for an extended component with the same velocity as the cloud was found. In the median, the peak brightness temperature of a cloud in the VGPS data is $\sim 20$ times brighter than the wing of the Galactic H I profile at the velocity of the cloud as determined from the average GBT spectra in the off positions. This indicates a large contrast between the clouds detected in the VGPS and any extended H I component with the same line-of-sight velocity. It should be noted that clouds with a large brightness contrast are easier to detect, so this number does not necessarily apply to the general population of fast-moving H I clouds.

H I column density maps were derived by summing over channels within $\pm 1.25 \Delta v$ (three times the Gaussian velocity dispersion) of the central velocity of the cloud. Figure 4 shows a selection of column density maps representative for the sample. Many clouds are compact, isolated H I features, such as $25.48+0.16+165$ and $43.08+0.92+112$. For the latter, there is some detached emission 0.25 from the brightest part, which is not listed as a separate cloud. The cloud $46.71+1.59+111$ is well resolved into a structure with multiple compact components. The most complicated structure is seen near longitude $29^{\circ}$ (upper right panel in Figure 4). The emission is distributed over $\sim 1^{\circ}$. The emission near the center of the image was listed as $28.76+0.58+142$. The emission in the lower part of this panel is part of $29.09+0.18+137$ and $28.69-0.09+132$. The willing eye could divide the structure seen in this part of the survey into more clouds than those listed in Table 1 . This was not 
done because we aim to avoid counting substructure as individual clouds. This issue will be discussed in more detail in Section 4.

Table 2 lists derived parameters of the clouds in the sample. The distance adopted is $d=R_{0} \cos l$ with $R_{0}=8.5 \mathrm{kpc}$, which assumes that each cloud is at the tangent point. This is a good assumption on average, but the uncertainty in the distance of an individual cloud can be large. A discussion of the error in the distance is deferred to Section 5. A lower limit to the random component of the velocity of the clouds is given by the parameter $V_{\text {pec }} \equiv V_{\mathrm{LSR}}-V_{\text {tan }}$ where the terminal velocity, $V_{\mathrm{tan}}$, is derived from the observations of ${ }^{12} \mathrm{CO}$ emission in the plane by Clemens (1985). This definition of $V_{\tan }$ takes advantage of the small random velocities of molecular clouds to infer Galactic rotation, while including information on the regular variations observed in the tangent-point rotation curve. The effect on this analysis of possible non-circular motions was tested by examining the dynamical simulations of Weiner \& Sellwood (1999), which include the effects of perturbations caused by a Galactic bar. Over the longitudes considered here the highest $V_{\mathrm{LSR}}$ occurs typically within $5 \%$ of the distance to the tangent point, and the largest displacement is only $20 \%$. It appears that the identification of the highest velocity in a $\mathrm{H}$ I spectrum as arising from the geometric tangent point is quite robust in the first galactic quadrant outside the galactic center. The derived values of $V_{\text {pec }}$ are lower limits in the case that the cloud is at a distance other than the tangent point.

Cloud dimensions were calculated from the angular sizes in Table 1 deconvolved with the $1^{\prime}$ VGPS beam assuming a Gaussian shape. The deconvolved angular size was larger than $1^{\prime}$ for all clouds. Formally all clouds in the sample are resolved by the $1^{\prime}$ beam, but some clouds are only marginally resolved. The H I number density $\left\langle n_{\mathrm{HI}}\right\rangle$ (Table 2 ) is defined as the peak column density divided by the harmonic mean of the major and minor dimensions of the cloud. The line width of the cloud provides an upper limit to the temperature of a cloud because the thermal width cannot exceed the observed width of the line profile. This leads to the upper limit $T_{\text {kin }} \leq 22 \Delta v^{2}$, with $T_{\text {kin }}$ in K and $\Delta v$ the FWHM width of the line profile in $\mathrm{km} \mathrm{s}^{-1}$. The density and limits to the temperature in Table 2 refer to a line-of-sight through the brightest part of the cloud. For the larger clouds $59.67-0.39+60$, and $60.70+1.02+58$, average values for several positions across the clouds were used. 


\section{Selection Effects}

\subsection{Velocity range}

Clouds may occur anywhere along the line-of-sight, but only those clouds with large forbidden velocities are counted. If a cloud is located in distance far from the tangent point, it must have a large random velocity component along the line-of-sight to be observed at a forbidden velocity. A cloud near the tangent point may have a smaller random velocity component along the line-of-sight in order to be observed at the same forbidden velocity, as

illustrated in Figure 5. Consider a velocity $V_{\mathrm{p}}$ with $V_{\mathrm{p}}<V_{\mathrm{tan}}$. In the first Galactic quadrant the velocity $V_{\mathrm{p}}$ will arise from more than one location along the line-of-sight. Clouds at those locations have a line-of-sight velocity distribution which is represented by a Gaussian in Figure 5. Some of these clouds appear at velocities larger than the tangent point velocity $V_{\tan }$ as indicated by the shaded area in Figure 5. If $V_{\mathrm{p}}$ is much smaller than $V_{\tan }$, only a small fraction of the clouds at the locations corresponding to $V_{\mathrm{p}}$ appear at forbidden velocities. This creates a bias for clouds observed at forbidden velocities to be located around the tangent point. The strength of this bias depends on the kinematic properties of the cloud population and on the direction of the line-of-sight. The total number of clouds at forbidden velocities is found by integration over all $V_{\mathrm{p}}$.

The velocity range of the search area is limited on the high-velocity side by the edge of the spectral band of the data, and on the low-velocity side by Galactic H I emission at permitted velocities. The high end of the velocity range extends far beyond the terminal velocity of Galactic HI. The velocity range of the search was extended to include the area sampled only by the high-velocity receiver channel of the VGPS VLA data. The absence of clouds detected near the upper velocity boundary of the survey (Figure 1) indicates that the velocity coverage of the survey does not introduce a significant selection effect in the number of clouds detected. It is found that the number of clouds decreases rapidly as the velocity increases beyond the tangent point velocity.

On the low-velocity side, a limit must be set to identify clouds with a large velocity beyond the terminal velocity. The actual maximum permitted velocity depends in a complicated way on Galactic longitude because of non-circular orbits of the gas and streaming motions associated with spiral arms. The effect of a spiral arm on the maximum permitted velocity becomes important if the spiral arm is close to the tangent point. A relatively large number of HII regions and supernova remnants associated with the spiral arm may be located close to the terminal velocity at the longitude of a spiral arm tangent. Disturbances of the interstellar medium by these objects are more likely to produce emission at forbidden velocities at these longitudes. Various tracers of the spiral arm tangents compiled 
by Englmaier \& Ortwin (1999) show that within the VGPS area, the line-of-sight becomes a tangent to the Sagittarius arm at $l \approx 51^{\circ}$ and to the Scutum arm at $l=24^{\circ}$ to $l=30^{\circ}$. We note that half of the sample of clouds in Figure 1 appears in the longitude range of the Scutum arm tangent. However, the remaining half of the sample does not appear near a spiral arm tangent.

The lower velocity limit of the cloud search $\left(V>V_{2 \mathrm{~K}}\right)$ is defined by the data, not by a model for Galactic rotation. This definition of the lower velocity limit of the search area takes into account the variations of the terminal velocity associated with spiral arms, and thus ensures homogeneous selection criteria for all longitudes. The lower velocity limit of the search area can be expressed in approximation in terms of $V_{\tan }$ by noting that for longitudes larger than $\sim 30^{\circ}, V_{2 \mathrm{~K}} \approx V_{\tan }+25 \mathrm{~km} \mathrm{~s}^{-1}$. The peculiar velocity along the line-of-sight, $V_{\mathrm{pec}}$, was determined relative to the maximum velocity of molecular clouds at the same longitude, which is determined more accurately than the H I terminal velocity.

\subsection{Survey area and geometric selection effects}

The VGPS survey area covers the first Galactic quadrant from $l=18^{\circ}$ to $l=67^{\circ}$. The latitude coverage of the VGPS is at least $\pm 1^{\circ} .3$, but increases to $\pm 1^{\circ} .9$ from longitude $46^{\circ}$ to $59^{\circ}$, and to \pm 2.3 from longitude $59^{\circ}$ to $67^{\circ}$. An outline of the survey area was given by Taylor et al. (2002) and Lockman \& Stil (2003). The varying latitude coverage of the survey must be taken into account in the analysis of the Galactic distribution of the clouds. The importance of the selection effect of the varying latitude coverage depends in part on the distribution of the clouds in the Galaxy. Clouds in the sample tend to be located near the tangent point (Section 4.1). The mean distance and Galactocentric radius of clouds in the sample vary accordingly with longitude. The larger distance of the tangent point at low longitudes compensates for the smaller latitude coverage of the survey, as illustrated by the following example. For a constant cloud scale height, at $l=25^{\circ}$, the maximum vertical distance at the tangent point probed by the search for clouds is $\pm 182 \mathrm{pc}$, while at $l=60^{\circ}$ it is $\pm 156 \mathrm{pc}$. If the scale height of the cloud population is much less than $100 \mathrm{pc}$, most of the clouds will be located within the survey area at all longitudes. The effect of the varying latitude coverage of the survey would be small in this case. However, if the scale height of the cloud population is much more than $200 \mathrm{pc}$, the fraction of the scale height probed by the VGPS is larger at low longitudes than at high longitudes. Thus, for a large cloud scale height, a constant number of clouds per unit volume would produce fewer clouds in the VGPS at high longitude, despite the larger latitude coverage there.

This argument assumes that all clouds are located at the tangent point. In fact, clouds 
detected at forbidden velocities occur along a range of distances along the line-of-sight, depending on the cloud-cloud velocity dispersion. Each positive velocity can arise from two locations (Figure 6). If the cloud-cloud velocity dispersion is large, the difference in distance between the near and far locations can be significant, and the volume probed at the far side is correspondingly larger. At higher longitudes $\left(l \gtrsim 50^{\circ}\right)$, clouds in the outer Galaxy may become visible at positive forbidden velocities. These clouds are always located behind the tangent point. In this case, the assumption that the distance of a cloud is on average the distance to the tangent point, breaks down. However the error introduced by assuming a tangent point distance remains negligible compared with the uncertainty in the distance of an individual cloud associated with the large random component of the line-of-sight velocity.

Interpretation of the relative numbers of clouds seen at high and low longitude requires careful modeling of the geometric selection effects related to the survey area and the distribution and kinematics of the ensemble of fast-moving clouds. Such models will be presented in Section 5.

\subsection{Identification of clouds}

Reliably identifying compact, faint emission in a large dataset such as the VGPS requires limits to avoid spurious detections from random fluctuations of the noise. Candidate detections were required to have a peak brightness temperature more than $10 \mathrm{~K}$, and 5 consecutive channels above $6 \mathrm{~K}$ (3 times the r.m.s. noise per channel). Cloud candidates were confirmed by follow-up observations with the GBT, except for the two larger clouds near longitude $60^{\circ}$. The sample presented here is believed to be complete within the specified limits of the search.

Several clouds in the sample appear as compact, isolated objects in the VGPS images. Other clouds are resolved, and may appear as more than one component. In the present search, multiple features with nearly the same velocity (compared with the line width of the brightest component) are considered to be a part of the same cloud. This approach aims to avoid a subjective subdivision of related emission into "sub-clouds", which could bias the analysis of the Galactic distribution of these clouds addressed in this paper.

Occasionally, HI emission is found which appears as a localized broad wing of the Galactic H I profile. Two approximately circular areas of $\sim 1^{\circ}$ in diameter were identified in the VGPS data, and are shown in Figure 7a,b. The structure at longitude $l=25^{\circ}$ is also

visible in data from the Leiden-Dwingeloo survey (Hartmann \& Burton 1997) shown by Koo \& Kang (2004). These structures are resolved into a number of smaller components in the 
arcminute-resolution VGPS images. Although some of the substructure would qualify as a "fast-moving cloud" according to our selection criteria, these structures were not included in the analysis of this paper. This is because these larger structures appear as wings on the Galactic line profile, not as objects with a distinct, well-defined forbidden velocity. The feature at $l \approx 25^{\circ}$ has a mass $M_{\mathrm{HI}}=5 \pm 2 \times 10^{4} \mathrm{M}_{\odot}$, assuming a tangent point distance. The mass of the feature at $l \approx 31^{\circ}$ is $M_{\mathrm{HI}}=4 \pm 1 \times 10^{4} \mathrm{M}_{\odot}$ assuming a tangent point distance. The main uncertainty in the mass determination is confusion with emission at permitted velocities. Broad wings of the Galactic H I line may be associated with supernova remnants or stellar wind bubbles with a large expansion velocity (Koo et al. 1990; Koo \& Heiles 1991). The structures in Figure 7a,b do not have a counterpart in the VGPS continuum images, but this does not exclude the possibility that these wings originate from old supernova remnants which are no longer visible in the continuum (Koo \& Kang 2004).

In this paper we concentrate on the small isolated clouds seen at forbidden velocities, which may or may not be related to the broad HI wings associated with old supernova remnants. We note that the cloud $25.48-0.16+165$ appears in the direction of the larger structure at $l \approx 25^{\circ}$. It is listed as an isolated cloud because of its much larger velocity, but it is possible that this cloud is associated with the larger structure.

\section{Galactic distribution of the clouds}

The distribution of the clouds in longitude and latitude is shown in Figure 8. The longitude distribution is most sensitive to a variation of the number of clouds with distance from the Galactic center. The latitude distribution is most sensitive to the scale height of the ensemble of clouds. The observed distributions are also affected by observational selection effects discussed in Section 4.

The longitude distribution in Figure 8 peaks near longitude $\sim 30^{\circ}$. The latitude distribution of the clouds is nearly flat. To use these data to constrain the Galactic distribution of the ensemble of clouds, the observed distributions were compared with simple models of the space distribution of the clouds integrated along the line-of-sight, including observational selection effects.

The Galactic distribution of the clouds is modeled as a layer with a surface density (number of clouds $\mathrm{kpc}^{-2}$ ) which may vary with Galactocentric radius, and a scale height and cloud-cloud velocity dispersion which do not vary with position. The scale height and velocity dispersion are treated as independent parameters because we do not wish to impose an assumption of hydrostatic equilibrium on the models by coupling the cloud-cloud velocity 
dispersion to a scale height.

Only a fraction of a population of fast moving clouds along the line-of-sight will appear at forbidden velocities. This fraction depends on the kinematic properties of the ensemble of clouds, and on the direction of the line-of-sight. The random line-of-sight velocity components of the clouds are expressed in the form of a probability distribution $f(v)$ with a single cloud-cloud velocity dispersion. Assuming an axially symmetric distribution of clouds $n_{c}(R)$ and a flat Galactic rotation curve $\left(R_{0}=8.5 \mathrm{kpc}, V_{0}=220 \mathrm{~km} \mathrm{~s}^{-1}\right)$ with circular orbits, the number density of clouds at velocity $V_{\mathrm{p}}$ can be expressed as a function of velocity $n_{c}\left(V_{\mathrm{p}}\right)$. Let $\Phi\left(V_{\mathrm{p}}\right)$ be the volume of space which corresponds with the velocity interval $\left\langle V_{\mathrm{p}}, V_{\mathrm{p}}+d V_{\mathrm{p}}\right\rangle$. $\Phi\left(V_{\mathrm{p}}\right)$ is a complicated function which contains details of the latitude coverage and geometrical selection effects. With these assumptions, the number of clouds with a line-of-sight velocity exceeding a limiting velocity $V_{\lim }$ at a particular longitude $l$, is

$$
N_{c}(l)=\int_{-\infty}^{V_{\tan }} n_{\mathrm{c}}\left(V_{\mathrm{p}}\right) \Phi\left(V_{\mathrm{p}}\right)\left\{\int_{V_{\lim }}^{\infty} f\left(v-V_{\mathrm{p}}\right) d v\right\} d V_{\mathrm{p}}
$$

The velocity distribution of the population of clouds thus acts as a weighting function in the integral of the number of clouds along the line-of-sight. The lower limit of the outer integral should take into account that at higher longitudes in the VGPS survey area clouds located outside the solar circle $\left(R>R_{0}\right)$ may in principle be seen at positive forbidden velocities. Little is known about the shape of the velocity distribution $f(v)$; we assume it is a Gaussian. The effect of replacing $f(v)$ with a function with non-gaussian wings (Siluk \& Silk 1974) is negligible for the current data.

The sample was selected with $V_{\lim }=V_{2 \mathrm{~K}} \approx V_{\mathrm{tan}}+25 \mathrm{~km} \mathrm{~s}^{-1}$. Inserting $V_{\mathrm{lim}}=V_{\tan }$ changes the normalization of the distribution $N_{c}(l)$ significantly, and hence the total number of clouds, but the shape of the distribution remains virtually the same for the longitude range covered by the VGPS.

The value of the cloud-cloud velocity dispersion is not well constrained by the data. The number of clouds at the tangent point velocity cannot be determined because of confusion with emission at permitted velocities. However, the distribution of the clouds in longitude and velocity can provide a plausible range for the value of the velocity dispersion. Velocity crowding at the tangent point velocity projects a large faction of the line-of-sight into a small velocity range. As a first approximation, each cloud can be assumed to originate from a location with (nearly) the tangent point velocity. If the cloud-cloud velocity dispersion were significantly larger than $50 \mathrm{~km} \mathrm{~s}^{-1}$, more clouds with peculiar motions in excess of $50 \mathrm{~km} \mathrm{~s}^{-1}$ would have been found. Also, the broad distribution of clouds in velocity compared with the wing of emission at permitted velocities suggests that the cloud-cloud velocity dispersion is larger than $\sim 10 \mathrm{~km} \mathrm{~s}^{-1}$. The range of the cloud-cloud velocity dispersion adopted in the 
sequence of models is $20 \mathrm{~km} \mathrm{~s}^{-1}$ to $50 \mathrm{~km} \mathrm{~s}^{-1}$ in steps of $10 \mathrm{~km} \mathrm{~s}^{-1}$. This range covers the velocities of clouds in the solar neighbourhood seen in optical and H I absorption experiments.

The primary effect of a larger cloud-cloud velocity dispersion is to make a larger fraction of the ensemble of clouds visible at forbidden velocities. If the model distributions are normalized to the observed distribution, the number density of clouds $\mathrm{kpc}^{-3}, n_{c}$, and the cloud-cloud velocity dispersion, $\sigma_{\mathrm{c}-\mathrm{c}}$, are correlated to make the product $n_{c} \sigma_{\mathrm{c}-\mathrm{c}}^{0.5}$ approximately constant (see Appendix A). This is the quantity which is constrained by the data. Models in Figure 8 assume a cloud-cloud velocity dispersion $\sigma_{\mathrm{c}-\mathrm{c}}=30 \mathrm{~km} \mathrm{~s}^{-1}$. Models with a different value for $\sigma_{\mathrm{c}-\mathrm{c}}$ would give nearly identical scaled distributions, but imply a different number density $n_{c}$.

Two prescriptions for the variation of the surface density of clouds in the disk were considered. Class 1 models use an exponential function of Galactocentric radius with the radial exponential scale length as a free parameter. Class 2 models assume that the surface density of clouds follows the surface density of molecular gas in the Galactic disk according to Dame (1993). A class 1 model has three input parameters (scale length, scale height and cloud-cloud velocity dispersion). A class 2 model has two input parameters (scale height and cloud-cloud velocity dispersion). The total number of clouds is also a free parameter because of the scaling of the models to the observed distributions. The vertical distribution of clouds is represented by a Gaussian. The vertical scale height is defined as the location where the number of clouds drops to half the number in the mid plane. The models were integrated along the line-of-sight within the VGPS survey area, and integrated in latitude and longitude to obtain model longitude and latitude distributions.

A Kolmogorov-Smirnov (KS) test was used to test the significance of differences between the observed distributions and the models. A large number of models was created, representing wide ranges in the radial scale length, vertical scale height, and cloud-cloud velocity dispersion. Models were tested against the observed cloud distributions and rejected at the 99\% confidence level. The KS tests were done separately for the longitude and the latitude distributions. Class 1 models failed the KS test outside the range of radial scale-lengths 2.8 $\mathrm{kpc}$ to $\sim 8 \mathrm{kpc}$. The high end of this range is more sensitive to the assumed scale height and cloud-cloud velocity dispersion, because a large scale height and a large cloud-cloud velocity dispersion have a more noticeable effect at the high longitude end of the VGPS. Among class 1 models, those with a radial scale length of $\sim 4.5 \mathrm{kpc}$ fit the data best. None of the models of class 2 was rejected by the KS tests. The small size of the sample does not allow us to discriminate between class 1 models and class 2 models. The correspondence between class 2 models and the data is interesting, but not necessarily unique.

KS tests against the latitude distribution of the clouds lead to a lower limit for the scale 
height of the clouds. Class 1 and class 2 models with the same scale height are nearly indistinguishable in the predicted latitude distribution (Figure 8). Models assuming a Gaussian layer with scale height smaller than 180 to 200 pc were rejected. The precise value depends somewhat on the assumed radial scale length and the cloud-cloud velocity dispersion. Panels $\mathrm{B}$ and D in Figure 8 show the latitude distribution for models with the smallest scale height not rejected by the data $(180 \mathrm{pc})$, and for a scale height three times this lower limit. Models with a large scale height fit the observed latitude distribution better.

The best-fitting model distributions can in principle be used to estimate the number of fast-moving clouds in the Galaxy. The result of such an estimate is very uncertain because of the number of assumptions which has to be made. The largest uncertainties result from the correction factor $f_{b}$ for the number of clouds outside the VGPS latitude range (related to the unknown scale height of the ensemble of clouds), and the factor $f_{v}$ which corrects for the effect of the lower velocity cut-off $v>V_{2 \mathrm{~K}}$ on the total number of clouds (a strong function of the unknown $\sigma_{c-c}$ ). The uncertainty in these factors is much larger than the differences between class 1 and class 2 models. We adopt a scale height of 540 pc (HWHM), which is 3 times the lower limit to the scale height of the clouds. In this case we have $f_{b}=2.7$, which varies by a factor $\sim 3$ up or down in the range of scale heights from $180 \mathrm{pc}$ to $2 \mathrm{kpc}$. Assuming $\sigma_{\mathrm{c}-\mathrm{c}}=30 \mathrm{~km} \mathrm{~s}^{-1}$, we find $f_{v}=3.1$, which varies by a factor $\sim 3$ in the range $20 \mathrm{~km} \mathrm{~s}^{-1}<\sigma_{\mathrm{c}-\mathrm{c}}<50 \mathrm{~km} \mathrm{~s}^{-1}$. The class 1 model with radial scale length $4.5 \mathrm{kpc}$ results in a surface density of fast moving clouds in the solar neighborhood $(R=8.5 \mathrm{kpc})$ of $\sim 7$ clouds $\mathrm{kpc}^{-2}$. The uncertainty in this number is at least an order of magnitude because of the uncertainties in $f_{b}$ and $f_{v}$. Other factors, such as the radial scale length of the distribution and the question of whether an exponential profile accurately represents the Galactic distribution of the clouds, increase the uncertainty further. This surface density of clouds implies a mass surface density of $4 \times 10^{-4} M_{\odot} \mathrm{pc}^{-2}$ in the form of fast moving clouds in the solar neighborhood. This is only a small fraction of the $5 M_{\odot} \mathrm{pc}^{-2}$ surface density of H I in the Galactic disk (Dickey \& Lockman 1990). When comparing these numbers, it should be kept in mind that the search for fast-moving clouds in the VGPS data is limited to clouds with fairly high column densities. Fast-moving clouds with column densities below the sensitivity limit of the VGPS have been observed in absorption and in emission. It is not clear at this time which fraction of the total population of fast-moving clouds is detected in the VGPS.

We also compared the best fitting model cloud distribution with the distribution of known H II regions (Lockman 1989; Lockman et al. 1996) and supernova remnants (Green 2004) within the VGPS survey area. H II regions and supernova remnants represent the most common places where matter is deposited into the interstellar medium with large velocities. The class 1 model fits shown in Figure 8A,B were integrated along the line-of-sight and over 
all velocities to create the total model longitude and latitude distributions in Figure 9. The distribution of H II regions declines faster with longitude than the model. The comparison with the supernova remnants is less clear. The model with the smallest scale height of the clouds coincides with the observed latitude distribution of known supernova remnants. However, it should be noted that the lower limit to the scale height of the clouds determined here appears to be limited mostly by the latitude coverage of the VGPS - the true value is likely much higher.

\section{Discussion and conclusions}

We present a sample of small neutral hydrogen clouds discovered in the VGPS with velocities up to $\sim 60 \mathrm{~km} \mathrm{~s}^{-1}$ beyond the local terminal velocity. The distribution in longitude and velocity of the clouds (Figure 1) suggests that they follow Galactic rotation despite their large peculiar velocities. This connection with the disk indicates that these clouds are not compact high-velocity clouds, which do not follow Galactic rotation (e.g. De Heij et al. 2002). On average, the distance to the tangent point is a good estimate of the distance for each cloud. The uncertainty in the distance of each individual cloud is large $(2$ to $3 \mathrm{kpc}$ for $\sigma_{\mathrm{c}-\mathrm{c}}=30 \mathrm{~km} \mathrm{~s}^{-1}$ ), but we can characterize the median properties of clouds in the sample. The median H I mass is $60 M_{\odot}$, and the median diameter is 8 pc. The median central density of a cloud is $7 \mathrm{~cm}^{-3}$. Many clouds are not well resolved in the present data, so the actual density may be higher than listed in Table 2. The clouds in the sample show a significant spread around these median parameters.

Peculiar velocities of the clouds were determined with respect to the terminal velocity of molecular gas. As such, the peculiar velocities derived here are lower limits to the random components of the line-of-sight velocity of each cloud. If a cloud is not located at the tangent point, the actual random component of the line-of-sight velocity is larger. The velocity of a cloud with respect to the ambient medium will also have a component perpendicular to the line-of-sight which is not constrained by the data. The largest peculiar velocities in the sample are in the range 50 to $60 \mathrm{~km} \mathrm{~s}^{-1}$ (three clouds). For all clouds in the sample $V_{\text {pec }}$ is a velocity component parallel to the Galactic plane. Any mechanism which aims to explain the origin of these clouds should produce this large velocity component in the Galactic plane, even at very small distances from the mid plane.

We note that the presence of small clouds with high forbidden velocities implies that some small H I clouds seen at permitted velocities may be displaced in velocity by as much as $60 \mathrm{~km} \mathrm{~s}^{-1}$. Such clouds would not be noticed as peculiar, unless they appear at velocities with little confusing emission, such as inter arm regions. Clouds with forbidden positive velocities 
in the second Galactic quadrant (Higgs et al. 2001; Kerton et al. 2002) may be members of the same population of fast moving clouds. The models discussed in Section 5 show that distances to these clouds in the second and third quadrant are very poorly constrained.

The models presented in Section 5 allow an evaluation of the Galactic distribution of the clouds without assumptions about the distance to any individual cloud. A sequence of models was generated to predict the sky distribution of clouds in longitude and latitude, taking into account the selection criteria. The hypothesis that the observed cloud distribution could have been drawn from a particular model distribution was tested with a KS test. The KS test uses the cumulative distribution of observed positions, and does not depend on the binning of the data.

The models not rejected by the KS test suggest that the number of clouds decreases with distance from the Galactic center, and that the scale height of the cloud population is large. Models with a scale height less than 180 pc (HWHM) are rejected at the $99 \%$ confidence level. Much larger scale heights $(>1 \mathrm{kpc}$ ) fit the data better. Models which assume that the surface density of the clouds follows the distribution of molecular gas in the Galactic disk, or an exponential profile with a scale length of $\sim 4.5 \mathrm{kpc}$ fit the observed longitude distribution best. The difference between these models is not statistically significant. The radial models would easily be distinguished by observations with the VGPS sensitivity and resolution at longitudes less than $20^{\circ}$. Similar observations with a larger latitude coverage than the VGPS would be valuable to determine the scale height of these clouds.

The number of clouds in the disk declines with distance from the Galactic center in a similar way as tracers of massive star formation. However, the clouds have a much larger scale height than tracers of star formation such as known HII regions in the VGPS survey area (Figure 9). The connection of the clouds with a large scale height and massive star formation in the disk may be established through the galactic fountain model (Bregman 1980). However, this remains speculative, as the only connection between the clouds described here and star formation in the disk is that both seem to have approximately the same radial distribution. A significantly larger sample of fast moving clouds in the disk is required to confirm this result.

The clouds described here show a resemblance in properties to the H I clouds several hundred parsec from the mid plane described by Lockman (2002, 2003); Lockman \& Pidopryhora (2005). The masses, velocities, and line widths are similar between the samples. The latitude distribution of the clouds described here is consistent with a scale height of the order of $1 \mathrm{kpc}$. The higher column densities and smaller diameters for the clouds in the present sample may be a selection effect because of differences in resolution and sensitivity between the VLA and the GBT. The VGPS data may reveal only the compact, high-column 
density members of a larger population of fast-moving clouds, because of the limited sensitivity to low-column density HI. On the other hand, the lower resolution of the survey by Lockman (2002) may have diluted the compact high-column density clouds which are detectable in the VGPS. We speculate that all fast-moving clouds could be part of a single population which occurs throughout the disk and up into the halo. An interesting test for this hypothesis is whether the halo clouds display a similar distribution in longitude. Also, it is desirable to increase the size of the sample of detected fast-moving clouds in the disk. This requires a combination of high resolution and high sensitivity which can be achieved with the new ALPHA receiver at the Arecibo radio telescope.

\section{A. Correlation of number of clouds with velocity dispersion}

The line-of-sight velocity of H I at longitude $l$ in the Galactic plane, following a flat rotation curve with amplitude $V_{0}$ is

$$
\frac{V_{\text {los }}}{V_{0} \sin l}=\frac{1}{\sqrt{x^{2}-2 x \cos l+1}}-1=F(x)
$$

with $x=d / R_{0}$ and $d$ is the distance from the Sun. Write $V_{\text {los }}$ as a Taylor series near the tangent point (distance $x_{0}=\cos l$ and $F^{\prime}\left(x_{0}\right)=0$ ):

$$
\frac{V_{\text {los }}}{V_{0} \sin l} \approx F\left(x_{0}\right)+\frac{1}{2} F^{\prime \prime}\left(x_{0}\right)\left(x-x_{0}\right)^{2}+\ldots
$$

Substituting the tangent point velocity

$$
V_{\tan }=V_{0}(1-|\sin l|) \frac{\sin l}{|\sin l|}
$$

which is valid in both the first and fourth Galactic quadrant, we find

$$
V_{\mathrm{los}} \approx V_{\mathrm{tan}}+\frac{1}{2} V_{0} \sin l F^{\prime \prime}\left(x_{0}\right)\left(x-x_{0}\right)^{2}
$$

The second derivative of $F(x)$ evaluated at the tangent point is

$$
F^{\prime \prime}\left(x_{0}\right)=-\frac{1}{\sin ^{3} l}
$$

It can be shown that the third derivative $F^{\prime \prime \prime}(x)=0$ if $F^{\prime}(x)=0$, so the next term in the Taylor series is of fourth order near the tangent point. Figure 10 shows a graph of $V_{\text {los }}$ and the Taylor expansion following Equation (A1) for longitude $l=45^{\circ}$. 
The number of clouds on the sky $N_{c}(l)$ is proportional to the number density of clouds near the tangent point $n_{c}\left(x_{0}\right)$ and the path length $\Delta d$ along the line-of-sight over which clouds from a population with velocity dispersion $\sigma_{\mathrm{c}-\mathrm{c}}$ are visible at forbidden velocities. An implicit integration in the z-direction is assumed. We define $\Delta d$ as the line-of-sight distance corresponding with a velocity $V_{\text {los }}=V_{\tan }-\sigma_{\mathrm{c}-\mathrm{c}}$. In this case $\left|x-x_{0}\right| R_{0}=\Delta d / 2$. Inserting in Equation (A1) yields

$$
\Delta d=\sqrt{8} R_{0}|\sin l|\left(\frac{\sigma_{\mathrm{c}-\mathrm{c}}}{V_{0}}\right)^{\frac{1}{2}}
$$

and from this follows for $N_{c}(l)$

$$
N_{c}(l) \sim n_{c}\left(x_{0}\right)|\sin l| \sqrt{\sigma_{\mathrm{c}-\mathrm{c}}}
$$

Note that $n_{c}\left(x_{0}\right)$ is a function of longitude $l$, but only through the distribution of clouds with Galactocentric radius. When the radial distribution of clouds in the Galaxy is specified in the model, $n_{c}\left(x_{0}\right)$ is a fixed function of longitude. In practice, clouds with line-of-sight velocities just beyond the terminal velocity cannot be counted because of confusion with the wing of the line profile of $\mathrm{HI}$ at permitted velocities. This confusion creates an ambiguity in the determination of $\sigma_{\mathrm{c}-\mathrm{c}}$, and an underestimation of $N_{c}(l)$. The predicted $N_{c}(l)$ from the models was rescaled to match the number of clouds in the sample. The effect of this scaling is that $N_{c}(l)$ is approximately the same for models with different values of $\sigma_{\mathrm{c}-\mathrm{c}}$. At any given

longitude, a degeneracy exists between the normalization of $n_{c}\left(x_{0}\right)$ and $\sigma_{\mathrm{c}-\mathrm{c}}$. Neither of these quantities can be estimated from the data separately because of confusion with unrelated emission at the tangent point velocity. The factor $|\sin l|$ in equation (A2) has the effect that the number of clouds increases more strongly with $\sigma_{\mathrm{c}-\mathrm{c}}$ at higher longitude. This partially lifts the degeneracy if the longitude distribution of clouds is considered. However, the sample of clouds presented in this paper is far too small to constrain the number of clouds at high longitude to the level required.

Once the longitude distribution is calculated for one value of the cloud-cloud velocity dispersion $\sigma_{\mathrm{c}-\mathrm{c}}$, the distribution for another $\sigma_{\mathrm{c}-\mathrm{c}}$ is found to a good approximation through the scaling relationship in Equation (A2). This scaling is valid only in the first and fourth quadrants, and for reasonably small $\sigma_{\mathrm{c}-\mathrm{c}}$. The models show that this result is valid for the range of $\sigma_{\mathrm{c}-\mathrm{c}}$ considered.

\section{B. Acknowledgements}

The VGPS is supported by a grant to ART from the Natural Sciences and Engineering Council of Canada. The authors thank the anonymous referee for carefully reading the 
manuscript and for comments which helped improve the clarity of this paper.

\section{REFERENCES}

Bregman, J. N. 1980, ApJ, 236, 577

Burton, W. B., \& Gordon, M. A. 1978, A\&A, 63, 7

Clemens, D.P. 1985, ApJ, 295, 422

Dame, T. M. 1993, in 'Back to the Galaxy', ed. S.S. Holt and F. Verter, AIP Press: New York, p. 267

De Heij, V., Braun, R., \& Burton, W. B. 2002, A\&A, 392, 417

Dickey, J. M., \& Lockman, F. J. 1990, ARA\&A, 28, 215

Dwarakanath, K.S. 2004, Bull. Astr. Soc. India, 32, 215

Dwarakanath, K.S., Goss, W. M., Zhao, J. H., \& Lang, C. C. 2004, J. Astr. Ap., 25, 129

Englmaier, P., \& Ortwin, G. 1999, MNRAS, 304, 512

Green, D. A. 2004, A Catalogue of Galactic Supernova Remnants (2004 January version), Mullard Radio Astronomy Observatory, Cavendish Laboratory, Cambridge, UK (available on the World-Wide-Web at "http://www.mrao.cam.ac.uk/surveys/snrs/")

Hartmann D., Burton W.B. 1997, Atlas of galactic neutral hydrogen, Cambridge University Press

Higgs, L. A., Kerton, C. R., \& Knee, L. B. G. 2001, AJ, 122, 3155

Kalberla, P.M.W., Westphalen, G., Mebold, U., Hartmann, D., \& Burton, W.B. 1998, A\&A, 332, L61

Kerton, C. R., Knee, L. B. G., Higgs, L. 2002, in ASP Conf. Ser. 276, Seeing Through the Dust: The Detection of HI and the Exploration of the ISM in Galaxies, ed. A. R. Taylor, T. L. Landecker \& A. G. Willis, (San Francisco: ASP), 136

Koo, B.-C., Reach, W. T., Heiles, C., Fesen, R. A., \& Shull, J. M. 1990, ApJ, 364, 178

Koo, B.-C., \& Heiles, C., 1991, ApJ, 382, 204 
Koo, B.-C., Kang, J.-H. 2004, MNRAS, 349, 983

Kulkarni, S. R., \& Fich, M. 1985, ApJ, 289, 792

Lockman, F. J. 1989, ApJS, 71, 469

Lockman, F. J., Pisano, D. J., \& Howard, G. J. 1996, ApJ, 472, 173

Lockman, F. J. 2002, ApJ, 580, L47

Lockman, F. J., \& Stil, J. M. 2004, in ASP Conf. Ser. 317, Milky Way Surveys: The Structure and Evolution of Our Galaxy, ed. D. Clemens, R. Y. Shah, \& T. Brainerd, (San Francisco: ASP), 20 (astro-ph/0311047)

Lockman, F. J. 2003, IAU symp Vol. 217, 2004, astro-ph/0311217

Lockman, F. J., \& Pidopryhora, Y. 2005 in in ASP Conf. Ser. 331, Extra-Planar Gas, ed. R. Braun, (San Francisco: ASP), 59

Mohan, R., Dwarakanath, K.S., \& Srinivasan, G. 2004, J. Astr. Ap., 25, 185

Münch, G., \& Zirin, H. 1961, ApJ, 133, 11

Radhakrishnan, V., \& Srinivasan, G. 1980, J. Astr. Ap., 1, 47

Siluk, R. S., \& Silk, J. 1974, ApJ, 192, 51

Taylor, A. R., Stil, J. M., Dickey, J. M., McClure-Griffiths, N. M., Martin, P. G., Rothwell, T., Lockman, F. J. 2002, in ASP Conf. Ser. 276, Seeing Through The Dust: The Detection Of H IAnd The Exploration Of The ISM In Galaxies, ed. A. R. Taylor, T. L. Landecker, \& A. G. Wills (San Francisco: ASP), 68

Weiner, B.J., \& Sellwood, J.A. 2003, ApJ, 524, 112

Welsh, B. Y., Sallmen, S., \& Lallement, R. 2004, A\&A, 414, 261 
Table 1. Observed quantities

\begin{tabular}{ccccccc}
\hline \hline $\begin{array}{c}\text { ID } \\
l, b, V_{\mathrm{LSR}}\end{array}$ & $\begin{array}{c}V_{\mathrm{LSR}} \\
\left(\mathrm{km} \mathrm{s}^{-1}\right)\end{array}$ & $\begin{array}{c}\text { Peak } T_{\mathrm{b}} \\
(\mathrm{K})\end{array}$ & $\begin{array}{c}\Delta v \\
\left(\mathrm{~km} \mathrm{~s}^{-1}\right)\end{array}$ & $\begin{array}{c}\text { Peak } N_{\mathrm{HI}} \\
\left(10^{20} \mathrm{~cm}^{-2}\right)\end{array}$ & $\begin{array}{c}\text { angular diameter } \\
\left({ }^{\prime} \times{ }^{\prime}\right)\end{array}$ & $\begin{array}{c}\int S d v \\
\left(\mathrm{Jy} \mathrm{km} \mathrm{s}^{-1}\right)\end{array}$ \\
\hline $24.84-0.98+157$ & $157.3(0.7)$ & $10.0(0.9)$ & $16.5(1.9)$ & 3.9 & $5 \times 3$ & $9.7(2.9)$ \\
$25.48+0.16+165$ & $164.6(0.6)$ & $18.7(1.5)$ & $8.2(1.4)$ & 2.9 & $2.9 \times 2.0$ & $5.7(0.7)$ \\
$27.20-0.82+133$ & $133.0(0.1)$ & $16.8(1.0)$ & $5.6(0.5)$ & 2.3 & $9.1 \times 2.8$ & $5.0(0.3)$ \\
$28.27+1.05+128$ & $128.1(0.1)$ & $19.2(1.0)$ & $5.6(0.5)$ & 2.9 & $4.8 \times 2.4$ & $4.6(0.3)$ \\
$28.69-0.09+132$ & $132.3(0.1)$ & $22.5(1.0)$ & $5.6(0.2)$ & 2.6 & $2.0 \times 1.6$ & $1.7(0.3)$ \\
$28.76+0.58+142$ & $141.6(0.2)$ & $12.2(1.0)$ & $5.9(0.5)$ & 0.9 & $8.7 \times 3.3$ & $7.2(1.1)$ \\
$29.09+0.18+137$ & $137.0(0.3)$ & $11.0(1.0)$ & $7.5(0.7)$ & 1.2 & $6.5 \times 2.7$ & $2.4(0.4)$ \\
$30.62-0.57+143$ & $142.8(0.4)$ & $13.9(2.1)$ & $5.9(0.9)$ & 1.8 & $3.7 \times 2.0$ & $5.1(2.4)$ \\
$32.28-0.72+142$ & $142.1(0.4)$ & $8.4(1.3)$ & $5.0(0.9)$ & 0.8 & $4.0 \times 1.8$ & $1.3(0.3)$ \\
$36.33+0.76+116$ & $115.9(0.4)$ & $7.9(1.0)$ & $7.5(1.2)$ & 1.9 & $4.9 \times 3.8$ & $8.0(3.0)$ \\
$42.42+0.76+101$ & $101.4(0.2)$ & $10.6(1.0)$ & $5.0(0.5)$ & 1.4 & $2.2 \times 1.7$ & $1.3(0.2)$ \\
$42.46+1.31+105$ & $104.6(0.5)$ & $8.6(1.0)$ & $11.7(1.6)$ & 1.4 & $17 \times 6$ & $27(5)$ \\
$43.08+0.92+112$ & $111.6(0.3)$ & $9.7(1.2)$ & $4.7(0.7)$ & 1.3 & $3.0 \times 1.9$ & $2.9(0.5)$ \\
$43.36-0.36+97$ & $96.6(0.2)$ & $13.0(1.4)$ & $4.5(0.5)$ & 1.5 & $4.1 \times 2.9$ & $1.0(0.2)$ \\
$46.71+1.59+111$ & $111.0(0.2)$ & $12.3(1.1)$ & $5.4(0.5)$ & 1.4 & $11.8 \times 4.3$ & $3.2(0.6)$ \\
$59.67-0.39+60$ & 60 & 38 & 4.2 & 3.9 & $72 \times 12$ & $580(200)$ \\
$60.70+1.02+58$ & 58 & 23 & 3.4 & 2.3 & $20 \times 6$ & $49(10)$ \\
\hline
\end{tabular}

Table 2. Derived quantities

\begin{tabular}{|c|c|c|c|c|c|c|c|c|}
\hline ID & $\begin{array}{c}\mathrm{d} \\
(\mathrm{kpc})\end{array}$ & $\begin{array}{c}\mathrm{z} \\
(\mathrm{pc})\end{array}$ & $\begin{array}{c}V_{\mathrm{pec}} \\
\left(\mathrm{km} \mathrm{s}^{-1}\right)\end{array}$ & $\begin{array}{l}\text { diameter } \\
(\mathrm{pc} \times \mathrm{pc})\end{array}$ & $\begin{array}{c}\left\langle n_{\mathrm{HI}}\right\rangle \\
\left(\mathrm{cm}^{-3}\right)\end{array}$ & $\begin{array}{c}M_{\mathrm{HI}} \\
\left(M_{\odot}\right)\end{array}$ & $\begin{array}{c}E_{k} \\
\left(10^{48} \mathrm{ergs}\right)\end{array}$ & $\begin{array}{c}T_{\mathrm{kin}} \\
\left(10^{3} \mathrm{~K}\right)\end{array}$ \\
\hline $24.84-0.98+157$ & 7.7 & -131 & 57 & $11 \times$ & 17 & 135 & 4.4 & $<6.0$ \\
\hline $25.48+0.16+165$ & 7.7 & +22 & 55 & $6 \times 4$ & 20 & 80 & 2.4 & $<1.5$ \\
\hline $27.20-0.82+133$ & 7.6 & -109 & 29 & $20 \times 6$ & 7 & 68 & 0.6 & $<0.7$ \\
\hline $28.27+1.05+128$ & 7.5 & +137 & 22 & $10 \times 5$ & 13 & 61 & 0.3 & $<0.7$ \\
\hline $28.69-0.09+132$ & 7.5 & -11 & 26 & $4 \times 3$ & 24 & 23 & 0.2 & $<0.7$ \\
\hline $28.76+0.58+142$ & 7.5 & +76 & 36 & $19 \times 7$ & 3 & 95 & 0.2 & $<0.8$ \\
\hline $29.09+0.18+137$ & 7.4 & +23 & 34 & $14 \times 5$ & 5 & 31 & 0.4 & $<1.2$ \\
\hline $30.62-0.57+143$ & 7.3 & -72 & 32 & $8 \times 4$ & 11 & 64 & 0.7 & $<0.8$ \\
\hline $32.28-0.72+142$ & 7.2 & -90 & 42 & $8 \times$ & 5 & 16 & 0.3 & $<0.6$ \\
\hline $36.33+0.76+116$ & 6.8 & +90 & 32 & $9 \times 7$ & 7 & 87 & 0.9 & $<1.2$ \\
\hline $42.42+0.76+101$ & 6.3 & +84 & 31 & $4 \times 3$ & 13 & 12 & 0.1 & $<0.6$ \\
\hline $42.46+1.31+105$ & 6.3 & +144 & 35 & $31 \times 11$ & 4 & 250 & 3.1 & $<3.0$ \\
\hline $43.08+0.92+112$ & 6.2 & +99 & 47 & $5 \times 3$ & 11 & 26 & 0.6 & $<0.5$ \\
\hline $43.36-0.36+97$ & 6.2 & -39 & 32 & $7 \times 5$ & 8 & 9 & 0.1 & $<0.4$ \\
\hline $46.71+1.59+111$ & 5.8 & +160 & 50 & $20 \times 7$ & 4 & 25 & 0.6 & $<0.6$ \\
\hline $59.67-0.39+60$ & 4.3 & -29 & 30 & $90 \times 15$ & 3 & 2500 & 22 & $<0.4$ \\
\hline $60.70+1.02+58$ & 4.2 & +75 & 26 & $24 \times 7$ & 6 & 200 & 1.3 & $<0.3$ \\
\hline
\end{tabular}


Fig. 1.- Distribution of clouds in longitude and velocity. A + symbol marks clouds listed in Table 1. The overall H I brightness temperature is shown by contours at 5, 10, 20, 40, and $80 \mathrm{~K}$, and by a gray scale for $T_{\mathrm{b}} \leq 10 \mathrm{~K}$. The smooth curve marks the velocity of the tangent point for a flat rotation curve with $V_{\odot}=220 \mathrm{~km} \mathrm{~s}^{-1}$. The dashed line marks the boundary of the search area in velocity. The horizontal band at $V_{\mathrm{LSR}} \approx 120 \mathrm{~km} \mathrm{~s}^{-1}$ is the result of low-level interference in the zero spacing data. [This figure is available as a separate jpg file]
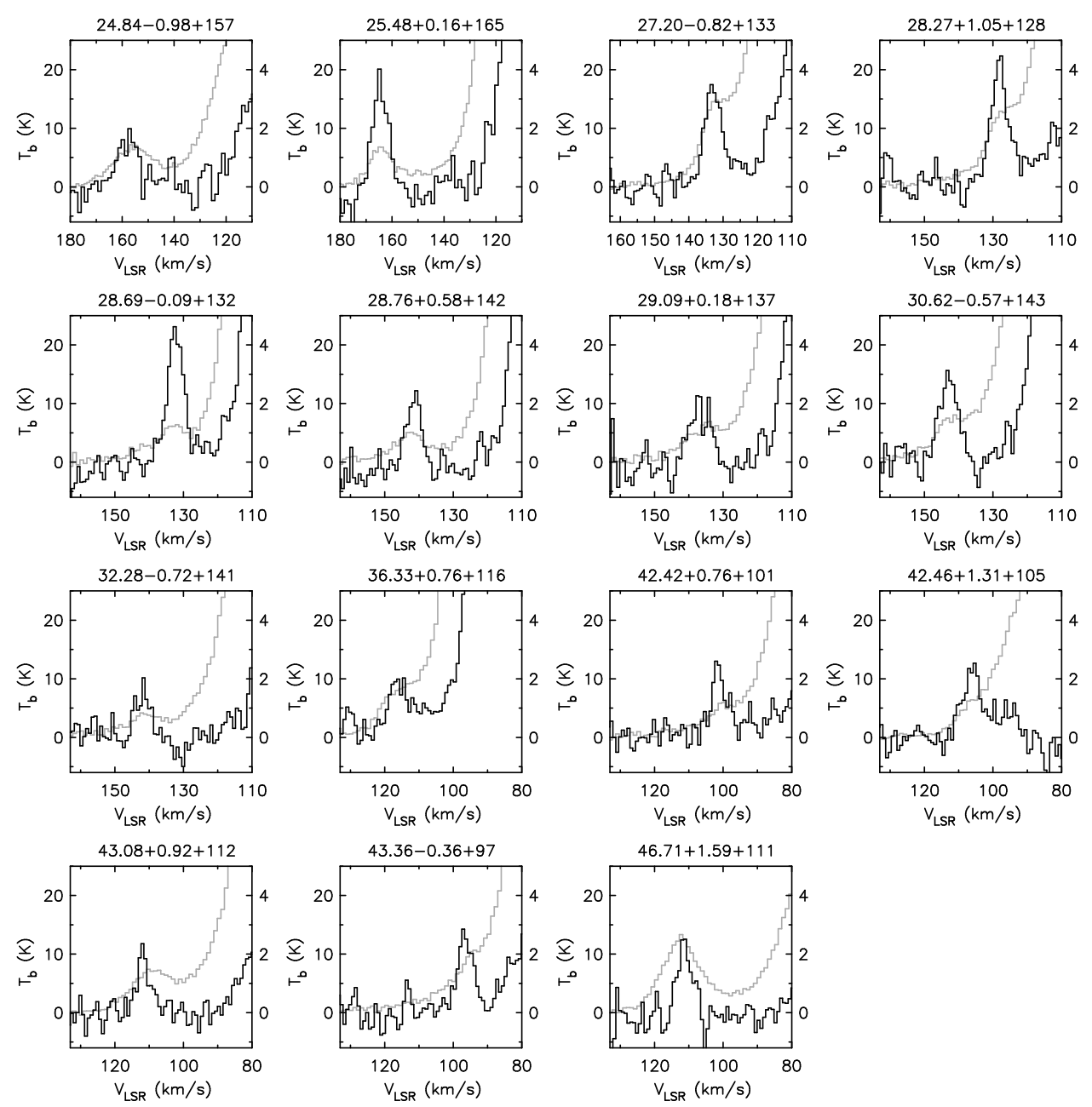

Fig. 2.- H I line profiles through the center of the clouds listed in Table 1. Dark lines are from VGPS data (temperature scale on the left axis), light lines from GBT data only (temperature scale on the right axis). 

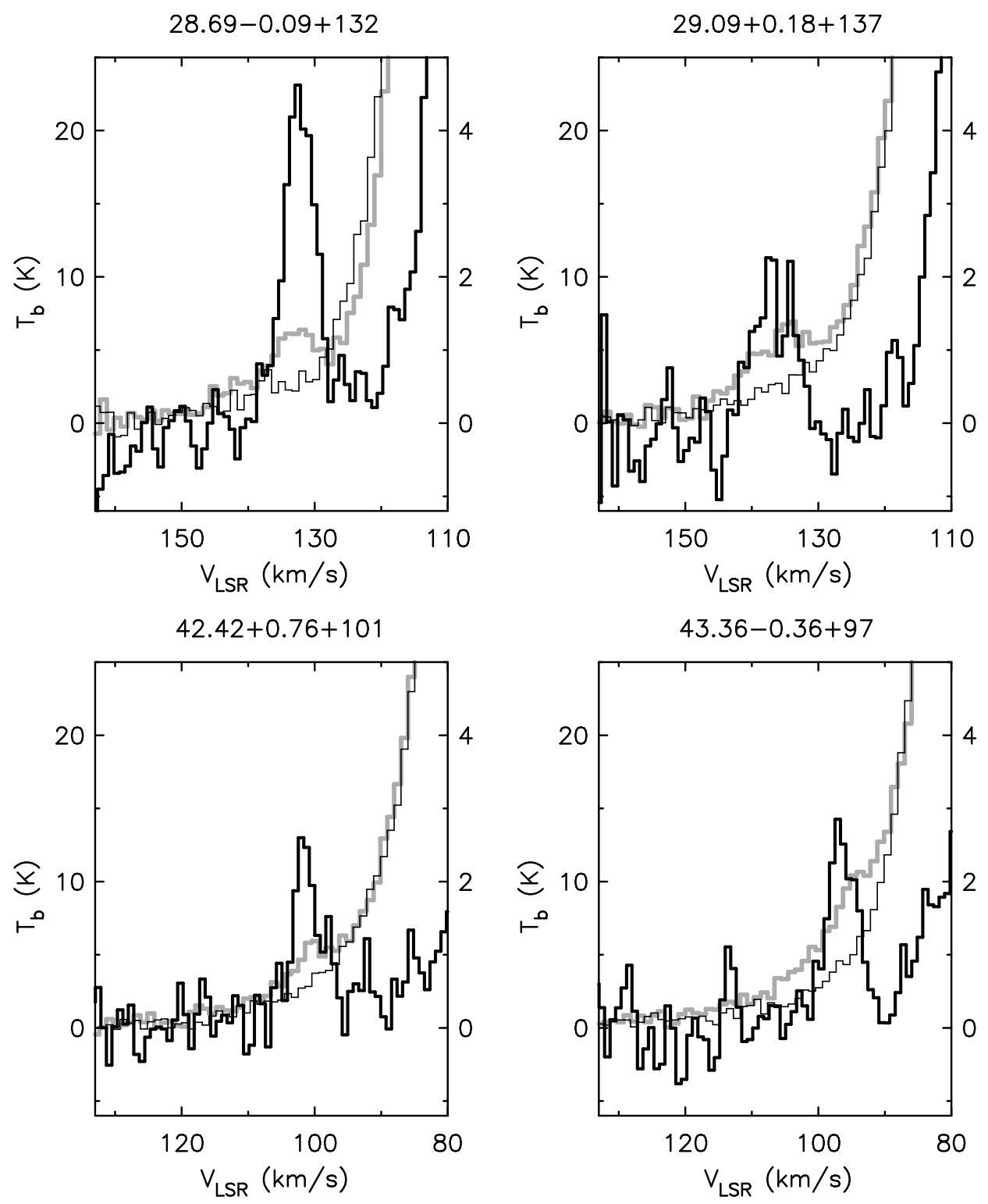

Fig. 3.- Enlargement of the line profiles of four clouds from Figure 2. The thick black histogram is the VGPS profile, the thick gray histogram is the GBT profile as in Figure 2. The thin black histogram shows the mean GBT profile of the off-cloud positions. The labels on the axes are the same as in Figure 2. 
Fig. 4.- H I column density maps of clouds listed in Table 1. Contours are drawn at $-3 \sigma$ (dashed), $3 \sigma, 4 \sigma, \ldots$ with $\sigma$ the r.m.s. noise in the map $(25.48+0.16+165: \sigma=$ $3.0 \times 10^{19} \mathrm{~cm}^{-2} ; 28.76+0.58+142: \sigma=1.5 \times 10^{19} \mathrm{~cm}^{-2} ; 43.08+0.92+112: \sigma=1.8 \times 10^{19} \mathrm{~cm}^{-2}$; $\left.46.71+1.59+111: \sigma=1.8 \times 10^{19} \mathrm{~cm}^{-2}\right)$. The gray scales are linear from $2.5 \times 10^{19} \mathrm{~cm}^{-2}$ to $3.0 \times 10^{20} \mathrm{~cm}^{-2}$ in all panels. [This figure is available as a separate jpg file]

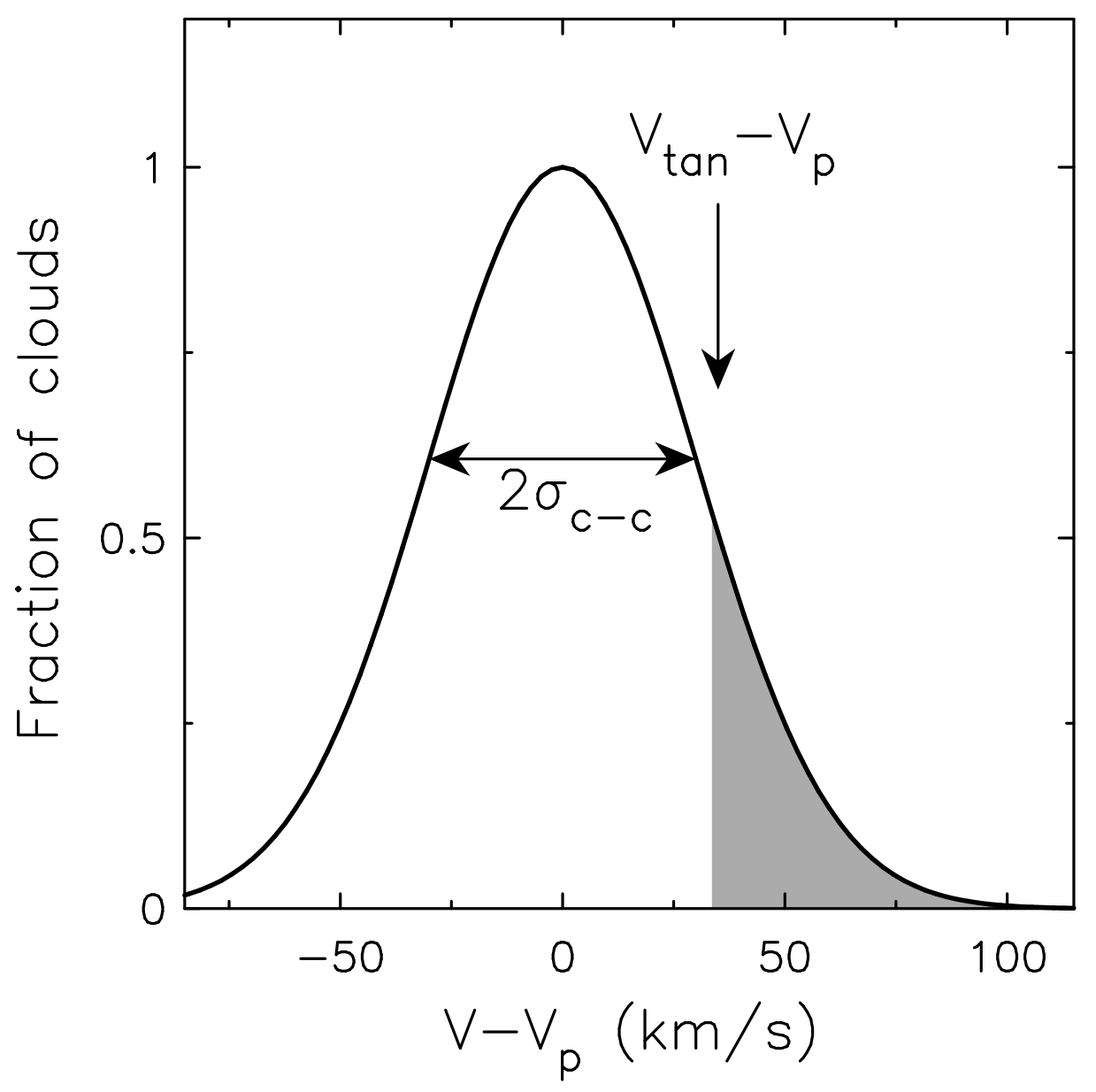

Fig. 5.- The line-of-sight velocity distribution of clouds at locations along the line-of-sight which correspond to a velocity $V_{\mathrm{p}}$ according to Galactic rotation. The shaded area indicates the fraction of clouds oberserved at velocities $V>V_{\tan }$. This diagram illustrates the case for $l=27^{\circ}, \sigma_{c-c}=30 \mathrm{~km} \mathrm{~s}^{-1}$, and for clouds at distances 5.1 and $10.1 \mathrm{kpc}$ from the Sun. 


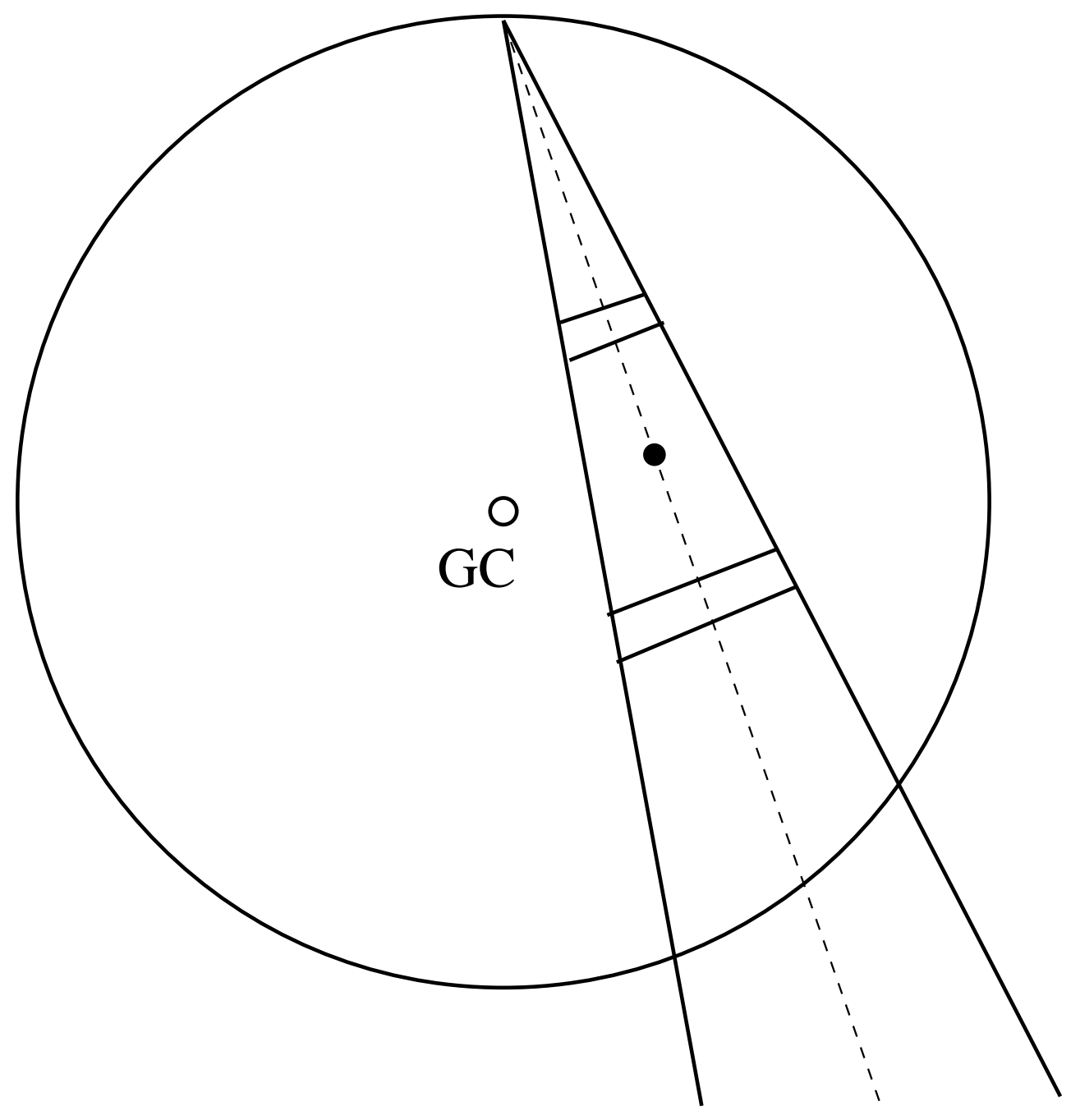

Fig. 6. - Illustration of the asymmetry of the volume probed at the near side and the far side of the tangent point, affecting the number of clouds observed at forbidden velocities. The circle indicates the orbit of the sun around the Galactic center (GC). The filled circle is the tangent point for the line-of-sight indicated by the dashed line. The near and far side volume elements shown here approximately represent locations with a velocity $30 \mathrm{~km} \mathrm{~s}^{-1}$ below the tangent point velocity. 
Fig. 7.- Wide-field H I column density maps of the Galactic plane with H I emission at forbidden velocities. Panels A and B show two approximately circular areas where the wing of Galactic H I is particularly broad. These regions were not included in the search for clouds. Panel $\mathrm{C}$ shows the clouds $59.67-0.39+60$ and $60.70+1.02+58$. Contrary to the structures in panels $\mathrm{A}$ and $\mathrm{B}$, these clouds do not appear as wings blended with the Galactic profile, but as separate objects. Gray scales indicate H I column density from $3 \times 10^{19} \mathrm{~cm}^{-2}$ to $3 \times 10^{20} \mathrm{~cm}^{-2}$, integrated over the velocity range of the feature. Panel A: Velocity range 128 to $156 \mathrm{~km} \mathrm{~s}^{-1}$. Panel B: Velocity range 122 to $149 \mathrm{~km} \mathrm{~s}^{-1}$. Panel C: Velocity range 55 to $65 \mathrm{~km} \mathrm{~s}^{-1}$. [This figure is available as a separate jpg file] 

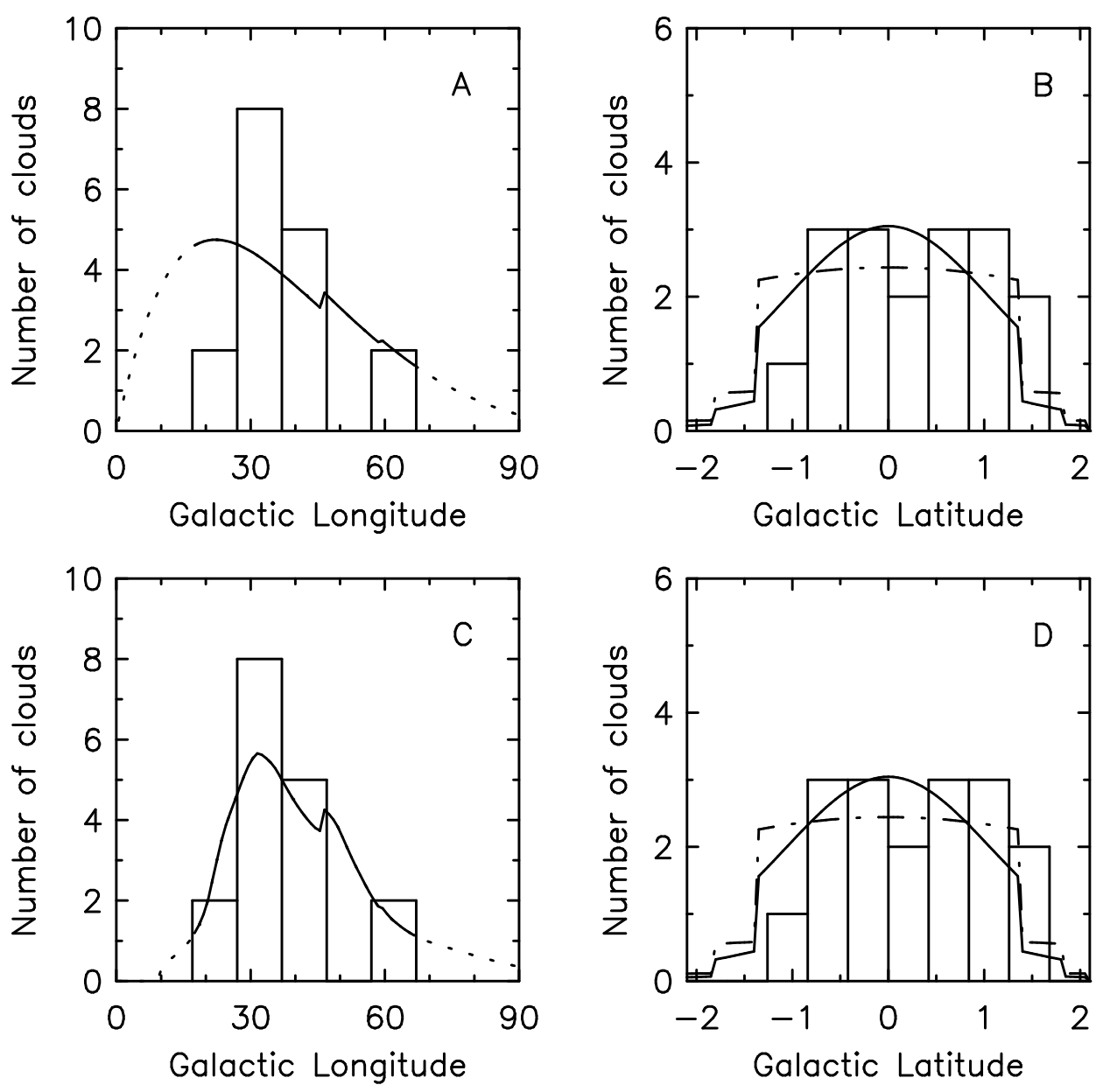

Fig. 8.- Distribution in longitude and latitude of the clouds compared with models for the spatial distribution of the clouds. Dotted curves show the model predictions outside the VGPS survey area. These parts were not included in the Kolmogorov-Smirnov tests. The models were scaled so that area under the model curves within the VGPS survey limits is the same as the area under the histograms. A: Model of class 1 in longitude with radial scale length $4.5 \mathrm{kpc}$, vertical scale height $180 \mathrm{pc}$, and $\sigma_{\mathrm{c}-\mathrm{c}}=30 \mathrm{~km} \mathrm{~s}^{-1}$. B: Latitude distribution of clouds compared with models of class 1 with vertical scale height $180 \mathrm{pc}$ (solid line) and $540 \mathrm{pc}$ (dot-dashed line). C: Model of class 2 in longitude, with scale height and $\sigma_{\mathrm{c}-\mathrm{c}}$ as in A. D: Latitude distribution of clouds compared with models of class 1 with vertical scale height 180 pc (solid line) and 540 pc (dot-dashed line). 

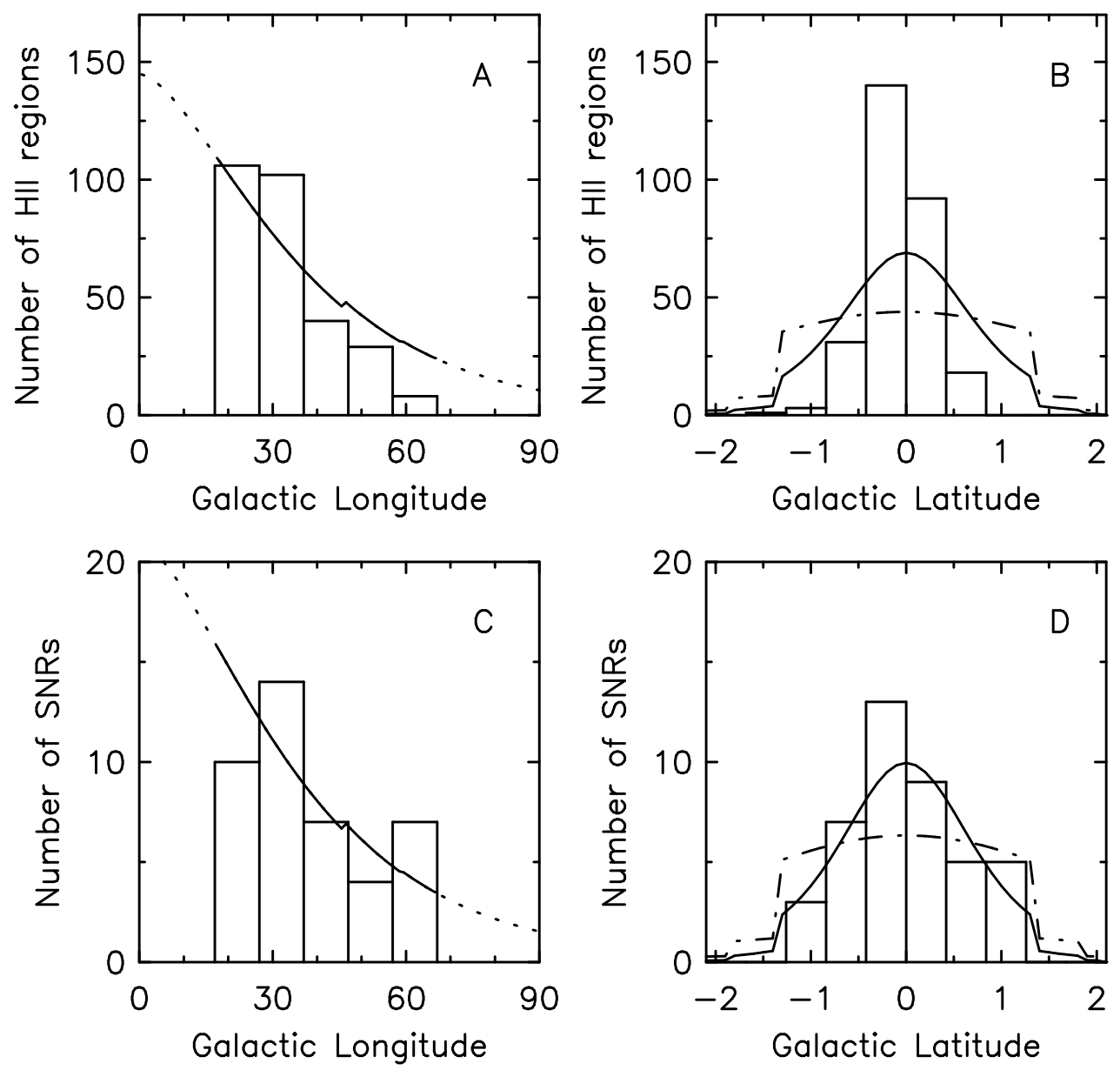

Fig. 9.- Distribution in longitude and latitude of Galactic H II regions and supernova remnants (histograms) and the model of class 1 shown in Figure 8A,B. The models were integrated over the line-of-sight without velocity weighting. 


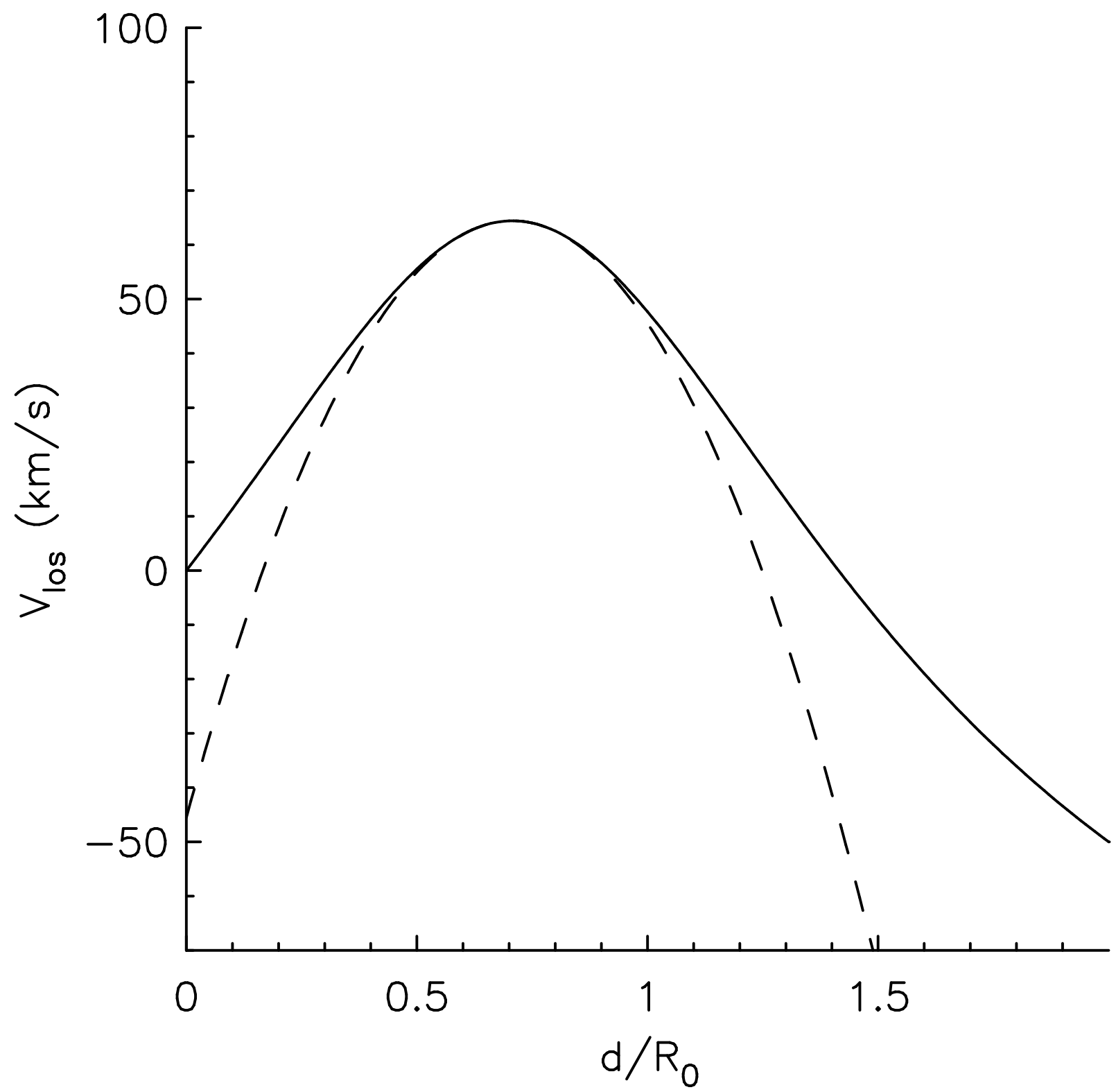

Fig. 10.- Relation between line-of-sight velocity and distance along the line-of-sight for longitude $l=45^{\circ}$. (solid curve). The Taylor expansion from Equation 1 at $l=45^{\circ}$ is shown as a dashed curve. 
This figure "f1.jpg" is available in "jpg" format from: http://arxiv.org/ps/astro-ph/0509730v1 
This figure "f4.jpg" is available in "jpg" format from: http://arxiv.org/ps/astro-ph/0509730v1 
This figure "f7.jpg" is available in "jpg" format from: http://arxiv.org/ps/astro-ph/0509730v1 